Service social

\title{
Service social des groupes en contexte multiculturel
}

\section{Ruby B. Pernell}

Volume 39, numéro 1, 1990

Le groupe ici et ailleurs

URI : https://id.erudit.org/iderudit/706455ar

DOI : https://doi.org/10.7202/706455ar

Aller au sommaire du numéro

Éditeur(s)

École de service social de l'Université Laval

ISSN

1708-1734 (numérique)

Découvrir la revue

Citer cet article

Pernell, R. B. (1990). Service social des groupes en contexte multiculturel. Service social, 39(1), 41-44. https://doi.org/10.7202/706455ar

\section{Résumé de l'article}

Ce court article est un commentaire aux propos de Kenneth L. Chau sur les expériences que vivent les membres des communautés culturelles en Amérique. L'auteure fait ressortir deux aspects de ce vécu, à savoir la couleur et l'adaptation, qui se traduisent, d'une part, par des difficultés tant personnelles que collectives au niveau de l'identité et de l'estime de soi et, d'autre part, par diverses stratégies d'adaptation, elles-mêmes sources de problèmes. Pernell réexamine les objectifs du service social des groupes en tenant compte du vécu des communautés ethniques et, par conséquent, des particularités qu'implique l'intervention en contexte multiculturel. 
Ruby B. Pernell, professeure, Case Western Reserve University, School of Applied Social Sciences, Cleveland, Ohio.

\section{Service social des groupes en contexte multiculturel*}

Ruby B. Pernell

Dans son article sur le service social des groupes en milieux multiculturels, Kenneth Chau identifie quatre expériences qui caractérisent le sens profond et la nature de la vie des membres des communautés culturelles en Amérique, soit :

- les déplacements et les pertes;

- les conflits culturels et l'adaptation;

- les préjugés de la population à leur endroit;

- le sentiment d'aliénation sociale et le sentiment d'être différents.

Ces expériences se déroulent généralement de la façon dont I'explique monsieur Chau; toutefois, son propos met essentiellement l'accent sur la communauté immigrante asiatique de première génération.

Je voudrais ici élargir le portrait qu'il présente en faisant ressortir deux aspects qui sont sources de problèmes : la couleur et l'adaptation. II n'y a pas de concept qui nous divise autant ou qui nous définisse mieux que la couleur. Nous sommes blancs ou noirs, bruns, jaunes, rouges. Celui qui est blanc appartient par conséquent à la classe dominante, simplement en vertu de sa couleur. Les autres couleurs définissent des groupes qui conservent des vestiges $d^{\prime} u n$ statut d'immigrant, peu importe depuis combien de temps ils habitent la région. Dans cette optique, même les Amérindiens sont des étrangers. Tous ces "gens de couleur " revivent de temps en temps les expériences décrites par monsieur Chau et doivent y faire face psychologiquement et socialement. 
Les Blancs qui arrivent d'Europe vivent eux aussi des expériences de déplacements et de pertes, des conflits de culture et d'adaptation, peut-être même des sentiments négatifs face à l'entourage, mais ils se mêlent à la population blanche dominante de sorte que, jusqu'à un certain point, leur "différence " s'estompe assez rapidement. Par contre, les "gens de couleur " portent leur différence sur le visage. Bien que par moments leur couleur passe inaperçue, ils peuvent rapidement se retrouver, avec ou sans avertissement, dans une situation où ils sont marginalisés, et c'est alors qu'ils se rendent compte que, dans une société raciste, ils ne sont jamais à l'abri du rejet.

En raison de leur différence de couleur, les Noirs d'Amérique sont sans cesse de nouveaux arrivants. Les plus petits changements, qu'il s'agisse de changements de quartier, de statut social ou économique, sont presque toujours pour eux source de stress. Dans leur lutte perpétuelle pour s'intégrer, ils n'arrivent pas à résoudre certains de leurs problèmes; certains points de jonction avec la communauté dominante demeurent chargés d'émotivité. Les gens leur font sentir qu'ils sont différents et, trop souvent, indésirables.

II est vrai que bon nombre de personnes et de familles " de couleur " semblent s'adapter plutôt bien à la société américaine, mais ils en paient le prix sous forme de stress psychologique. Par exemple, ils prennent conscience du fait que la société les confond à leur groupe ethnique, que l'individu représente son groupe et que le groupe représente l'identité de l'individu, expérience collective que les Blancs vivent rarement. Cette situation apporte aux membres des communautés ethniques des problèmes additionnels $d^{\prime}$ 'identité et d'estime de soi.

À force de subir les perceptions négatives des autres et, en même temps, la dévaluation de leur groupe ethnique, ces citoyens peuvent développer différentes stratégies d'adaptation, par exemple la confrontation et la revendication ou, au contraire, la passivité, ou encore une mise à l'écart volontaire par rapport à la société pour ainsi créer des sous-sociétés dans lesquelles ils sont plus susceptibles d'être acceptés et de retrouver statut et pouvoir.

Toutefois, il existe aussi de nombreux cas d'individus et de familles qui succombent au stress et à la dépression, d'hommes qui voient leur rôle ébranlé, des situations de pauvreté envahissante, de dépendance, de crime et de violence.

Tout ceci représente beaucoup de travail pour l'intervenant de groupe : agir à titre de facilitateur et de courtier dans la recherche d'un meilleur statut, aider les groupes à se donner les moyens de faire avancer leurs causes, lutter contre la passivité, inciter les personnes laissées pour compte et antisociales à s'impliquer socialement. À 
l'instar de Chau, je crois qu'il est très important d'offrir des groupes de prévention, de développement et de socialisation. Ce sont là des objectifs du service social des groupes que nous avons pratiquement abandonnés à un certain moment et qui refont surface devant les besoins actuels. II s'avère de nouveau pertinent de travailler avec les enfants en amalgamant travail social, récréation et éducation informelle puisque nous trouvons ainsi des façons d'éviter qu'ils soient entraînés par le réseau et la sous-culture des " gangs ».

Dans la communauté noire, l'homme noir soulève beaucoup d'inquiétudes. Des changements troublants sont apparus au cours des dernières années qui, à mon sens, reflètent un affaiblissement collectif de l'estime de soi : on cherche à bâtir un personnage qui, en apparence, accepte que son identité soit telle que la société la perçoit. Il s'agirait en somme d'un stéréotype qui se suffit à lui-même.

Quand Jesse Jackson exhorte un groupe de jeunes Noirs à crier "je suis quelqu'un ! ", cela indique clairement que le problème d'estime de soi n'est pas un problème individuel, mais bien un phénomène de groupe, qui s'est introduit dans le psychisme d'un peuple à la suite de messages que les membres, leurs parents et leurs grands-parents ont reçus de leur entourage immédiat et de l'ensemble de la communauté.

Lorsque nous nous attaquons à des problèmes de développement personnel et social, de survie et de croissance dans des conditions sanitaires adéquates, de cohésion et de support intragroupe, et de prise de pouvoir politique et économique dans un groupe ethnique distinct et stigmatisé de notre société multiculturelle, le besoin le plus criant, c'est le leadership. Dans le cas de la communauté noire, nous parlons de leadership noir. Le leadership de I'homme noir apparaît particulièrement important lorsque nous travaillons avec des groupes de jeunes Noirs. C'est un défi pour les travailleurs sociaux de groupe de recruter et d'entraîner des bénévoles, de trouver des façons originales de les mettre à contribution et de les superviser. C'est aussi un défi de développer les types de services de groupe qui répondent aux besoins diversifiés des Noirs dans notre société multiculturelle.

Qu'il s'agisse de Noirs ou d'autres gens de couleur, hommes ou femmes, les intervenants doivent être en mesure de comprendre les effets du racisme sur la personne et sur ses relations sociales immédiates. Ils doivent aussi savoir comment des mécanismes et des structures comme le système familial et les "gangs" évoluent en réaction aux effets combinés du racisme, du manque de pouvoir réel et de la valeur accordée par l'ensemble de la société au développement social et économique. 
Nous avons tendance à considérer l'accroissement du pouvoir (empowerment) comme l'objectif social majeur de notre travail avec les groupes. Je suggérerais un concept plus englobant : la libération. La libération de l'identité positive du Moi; la libération des stéréotypes négatifs qui envahissent l'âme; la reconnaissance et l'utilisation par la personne de son espace vital; la canalisation de l'énergie, par le biais d'une action collective, visant à faire tomber les barrières qui nous séparent. Enfin, cela implique de travailler à la libération de l'ensemble de la société, emprisonnée par les frontières de couleurs que ses membres ont eux-mêmes érigées en réaction aux revendications de leurs concitoyens. D'une certaine façon, nous sommes tous des immigrants qui aspirons à la liberté pour profiter pleinement des avantages d'une société multiculturelle.

Note

* Texte présenté au symposium de Montréal. Traduction de Lyne Champoux. 